\title{
Integration of Massage Therapy in Outpatient Cancer Care
}

\author{
Virginia S. Cowen, $\mathrm{PhD}, \mathrm{LMT}^{1}{ }^{1 *}$ Barbara Tafuto, $\mathrm{MLS}^{2}$ \\ ${ }^{1}$ Department of Primary Care, Rutgers University, School of Health Professionals, Newark, NJ, USA \\ ${ }^{2}$ Department of Health Informatics, Rutgers University, School of Health Professions, Newark, NJ, USA
}

Background: Massage therapy can be helpful in alleviating cancer-related symptoms and cancer treatment-related symptoms. While surveys have noted that cancer patients seek out massage as a nonpharmacologic approach during cancer treatment, little is known about the integration of massage in outpatient cancer care.

Purpose: The purpose of this study was to examine the extent to which massage is being integrated into outpatient cancer care at NCI-designated Cancer Centers.

Setting: This study used descriptive methods to analyze the integration of massage in NCI-designated Cancer Centers providing clinical services to patients $(n=62)$.

Design: Data were collected from $91.1 \%$ of the centers $(n=59)$ using content analysis and a telephone survey. A dataset was developed and coded for analysis.

Main Outcome Measure: The integration of massage was assessed by an algorithm that was developed from a set of five variables: 1) acceptance of treatment as therapeutic, 2 ) institution offers treatment to patients, 3 ) clinical practice guidelines in place, 4) use of evidence-based resources to inform treatment, and 5) shared knowledge about treatment among health care team. All centers were scored against all five variables using a six-point scale, with all variables rated equally.

Results: The integration of massage ranged from not at all (0) to very high (5) with all five levels of integration evident. Only 11 centers $(17.7 \%$ of total) rated a very high level of integration; nearly one-third of the centers $(n=22)$ were found to have no integration of massage at all-not even provision of information about massage to patients through the center website.

Conclusions: The findings of this analysis suggest that research on massage is not being leveraged to integrate massage into outpatient cancer care.

KEY WORDS: massage; integrative oncology; nonpharmacologic; cancer treatment-related symptoms; integration algorithm

\section{INTRODUCTION}

Cancer-related symptoms (CRS) and cancer treatment-related symptoms (CTRS) encompass an array of physical and psycho-emotional indications including discomfort, pain, fatigue, and anxiety. CRS and CTRS have potential to interfere with a patient's quality of life. ${ }^{(1-6)}$ For nonpharmacologic approaches to treat CRS/CTRS, a growing number of patients use massage and other complementary/alternative medicine (CAM) therapies. ${ }^{(7-11)}$ Research also suggests that massage can be effective in management of CRS/ CTRS specifically through the reduction of chronic pain, anxiety, fatigue, and mood disturbance, ${ }^{(12-16)}$ and that it can be safely integrated into outpatient cancer care. ${ }^{(17-21)}$ While there is an established field of research on massage for CRS/CTRS, the extent to which this is translational (i.e., applied in clinical care) $)^{(22)}$ is unclear.

The Institute of Medicine (IOM) identifies the translation of CAM from distinct practices into mainstream medical care through several levels. ${ }^{(23)}$

1. Dissemination of information: This can occur in many forms. On the lower end general educational materials can summarize theories and practices for a CAM therapy. A higher level of information includes a summary of the potential benefits a CAM therapy can have on physical symptoms, psychosocial outcomes, or general well-being.

2. Incorporation of a CAM therapy into care: This may involve CAM practitioners working alongside conventional health care providers or utilization of CAM therapy techniques by conventional health care professionals.

3. The use of evidence-based decision-making: This is rooted in review of research studies to ensures a basis for effectiveness of treatment in general, as well as comparative evaluation for treatment protocols.

4. Development of clinical practice guidelines: The creation of clinical practice guidelines establishes a treatment approach based on professional consensus that incorporates evidence for best practices, an essential part of oncology care. ${ }^{(24)}$ 
5. Shared knowledge: Documentation of massage in the patient's medical record, as a component of integration, provides opportunities to incorporate a CAM therapy into the patient's care plan. From a workflow aspect, this may involve coordination of patient appointments. ${ }^{(25,26)}$ From an outcomes aspect, shared knowledge promotes understanding of treatment effects among the members of the health care team. ${ }^{(27)}$ Inclusion of this information in each patient's electronic medical record (EMR) allows monitoring of effects of massage treatment on CRS/CTRS relative to other interventions. ${ }^{(28,29)}$

Ultimately, integrated care occurs when members of the health care team work in a coordinated manner to treat patients and monitor patient progress. The translation levels identified in the IOM report presents an opportunity to quantify integration of a CAM therapy into medical care. Analysis of the incorporation of massage into outpatient cancer care provides the opportunity to understand integration and explore the extent to which the body of research on massage for CRS/CTRS is leveraged.

The National Cancer Institute (NCI) classifies three different types of cancer centers: comprehensive, clinical, and basic laboratories. NCI-designated comprehensive and clinical Cancer Centers are considered to be cutting-edge, progressive institutions at the forefront of patient care. All NCI-designate Cancer Centers are considered leaders in clinical care, education, community outreach, and other critical components of cancer care. ${ }^{(30)}$ Comprehensive cancer centers receive an additional designation in recognition of a leadership role in research. (NCI basic laboratories are research centers that do not provide clinical care to patients.) A growing number of NCI-designated Cancer Centers s have established integrative oncology services for patients undergoing treatment, as well as through survivorship programs. (31) These initiatives recognize the potential for CAM therapies - including massage - to alleviate CRS/ CTRS. Since NCI-designated Cancer Centers are at the forefront of comprehensive cancer care, examination of the extent to which massage is integrated into outpatient cancer care is warranted. Previous research on NCI-designated Cancer Center websites indicated that massage is among the CAM therapies mentioned on cancer center websites. ${ }^{(31)}$ But little is known about the level of massage integration into patient care. The purpose of this study was to examine the extent to which massage is being integrated into outpatient cancer care.

\section{METHODS}

This study involved descriptive research of massage at NCI-designated Cancer Centers. A list of NCI-designated comprehensive and clinical cancer centers was compiled from the NCI online directory. Data were sourced from a systematic analysis of each center's website and a follow-up telephone survey.

All information about massage associated with outpatient care at NCI-designated Cancer Centers was sought in order to explore the integration of massage in outpatient care. The term "massage" is used in this study in a broad manner because massage includes an array of techniques and modalities ranging from energy therapies to vigorous manipulation of soft tissues. Because massage techniques may be used by various providers with therapeutic intentions, no $a$ priori determination was made to include or exclude modalities, settings, or providers of massage.

For each NCI-designated Cancer Center, a summative research approach was employed. The website for each center was systematically searched for massage-related information. This involved identification of relevant text, its extraction and interpretation, followed by coding into variables for analysis. The link to each NCI-designated Cancer Center's official home page was accessed using the NCI online directory. For each center, the primary search process included examination of sidebars, sitemaps, and drop-down menus from each. Any area of the website was considered potentially relevant if it included any of the following words or word combinations: massage, integrative medicine, integrative health, alternative medicine, complementary medicine, or CAM. All links with potentially relevant information were explored and available relevant text extracted. If any subsequent pages had links that were potentially relevant, those links were further explored and relevant text extracted. When information could be located using links, all linked areas of the website were explored until exhausted (i.e., no new information was revealed).

A secondary search was conducted if relevant information was not located via the primary search. The secondary search used a free text search for 'massage' through the general search box on the center's home page. All relevant links were explored and available text extracted. A tertiary search was attempted if the previous searches did not return any results. An Internet search was conducted using www.google.com with a search term combination of the NCI-designated Cancer Center Name and Massage. Any links to the NCI-designated Cancer Center website or affiliates were followed and examined. Links to external websites were excluded from this analysis.

All text containing information relevant to massage was extracted as unstructured data. Analysis and interpretation of the unstructured data was performed to identify themes and potential variables related to integration. A dataset was created using each NCIdesignated Cancer Center as a "case" and relevant content entered as unstructured text under broad categorical variables (e.g., modalities, requirements for patient referral). All searches were conducted by 
a single researcher. A random sample of searches was repeated by a second researcher for validity.

A follow-up survey was conducted to verify or supplement information from the website analysis. The massage provider, program, or department was contacted by telephone. Each respondent was asked to verify that the information extracted from the website was accurate, current, and comprehensive. Additional survey questions asked for information about referral requirements, clinical practice guidelines, and documentation for massage treatments. All telephone surveys were conducted by a single researcher. Data from the telephone survey were combined into the dataset in a three-step process: first, to fill gaps when information was not available through the website; secondly, to verify web-based data (in this case no changes were made); thirdly, to replace information when disagreement was evident between the website and survey sources.

The final dataset was coded using an inductive approach. ${ }^{(32)}$ All text was read by a single researcher in an iterative manner to identify concepts relevant to delivery of massage to patients and the integration of massage in patient care. These concepts were used to create broad categorical variables. The dataset - including these categorical variables - was then read by a second researcher. Additional categorical variables were identified. The dataset was then coded using these variables. Both researchers reviewed the coding to ensure agreement. Prior to the initiation of any study procedures, this project was reviewed and approved as an expedited protocol by the Rutgers University Biomedical and Health Sciences-Newark Human Subjects Institutional Review Board (Protocol \# Pro20150001821).

\section{RESULTS}

This study included all National Cancer InstituteDesignated Cancer Centers providing clinical services to patients $(n=62$ centers). Data were collected from $91.1 \%$ of the centers $(n=59)$. Fifty $(n=50)$ centers participated in the telephone survey, nine $(\mathrm{n}=9)$ centers (while not participating in the survey) had available public information on the website regarding massage services. Three $(n=3)$ of the centers neither participated in the survey nor provided information about massage services for cancer patients on their website. (See Table 1)

Massage specifically for cancer patients was offered at 34 NCI-designated Cancer Centers (54.8\%). A range of massage styles were represented (See Figure 1). Analysis of massage availability by type of center revealed a higher percent of comprehensive cancer centers offering massage: 28 comprehensive cancer centers $(62.2 \%$ of the total number of comprehensive cancer centers) compared to clinical cancer centers where massage was offered at only
Table 1. NCI-Designated Cancer Centers Included in Analysis

\begin{tabular}{|c|c|c|}
\hline & $N$ & $(\%)$ \\
\hline $\begin{array}{l}\text { Participated in the telephone survey and } \\
\text { accessible web-based information }\end{array}$ & 50 & $80.6 \%$ \\
\hline Web-based information available only & 9 & 14.5 \\
\hline Did not participate/no information available & 3 & 4.8 \\
\hline Total & 62 & 100.0 \\
\hline Offer massage to cancer patients & 34 & 54.8 \\
\hline
\end{tabular}

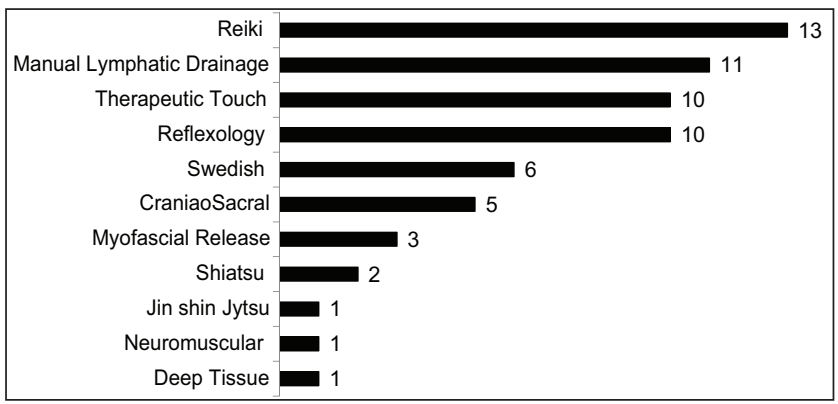

FIGURE 1. Number of centers offering massage styles.

$6(35.3 \%$ of the clinical cancer centers). There was evidence that massage therapists practiced with autonomy at the majority of the cancer centers because physician referral for oncology massage was required at only 10 centers $(29.4 \%$ out of the 34 centers offering massage). Physician referral was requested - but not required - by massage programs at three centers $(8.8 \%)$. At the remainder of the centers, scheduling a massage was up to individual patients and massage providers.

Centers were specifically queried on oncology massage guidelines. Formal clinical practice guidelines were in place at 16 centers $(47 \%)$. Of these centers, six $(37.5 \%)$ drew upon a combination of research literature and oncology massage textbooks; four (25\%) drew only upon research literature; and two $(12.5 \%)$ drew only upon textbooks. For the remaining four centers $(25 \%)$, sources for clinical practice guidelines included recommendations from conventional clinical providers or information obtained from an oncology massage course.

Documentation of massage treatment-related information was collected for pre- and post-massage treatment. Out of 34 centers offering massage, 28 centers $(82 \%)$ had some type of pre- or post-massage treatment documentation. This documentation included written information or entries into the patient's EMR. Pretreatment data were documented for 11 centers $(32.4 \%)$. Written health history forms were required at eight centers $(23.5 \%)$ prior to massage treatment, whereas two centers $(5.8 \%)$ require only a written questionnaire. A single center used both a health history form and a questionnaire. A pre-massage interview plus written documentation was completed at only eight centers $(23.5 \%)$. An additional 
12 centers conducted a pre-massage interview but did not indicate that any pre-massage information was documented in written form.

Pre-massage review of the patient's medical chart was available to the massage providers at 15 centers (44.1\%). Of these, 11 centers $(32.4 \%)$ did not document any additional written pre-massage information. In addition to the medical chart review, a written pre-massage health history was taken at four centers $(11.7 \%)$ This indicates that massage providers had access to patient health and treatment information. At 12 centers $(35.3 \%)$, massage providers did not take any written pre-massage information and did not have access to the patient's medical chart. This suggests that massage although offered is not considered part of the center's treatment protocol.

Documentation of some aspect for post-massage treatment was completed at 24 centers $(70.5 \%)$. A written summary of treatment was taken at seven centers $(20.6 \%)$. Of these, three centers submitted additional documentation in the patient's EMR and four centers had only a written summary but no access to insertion in the EMR. Documentation of massage only in the EMR but not in written form was completed at 17 centers $(34 \%)$. Massage providers at 10 centers $(29.4 \%)$ did not document any post-massage information either in the EMR or in written form.

While 28 centers $(46 \%)$ of the NCI-designated Cancer Centers did not offer massage to cancer patients, five of these centers acknowledged the therapeutic value of oncology massage either by publishing information about massage on the website $(n=1)$, referring to an outside entity for massage $(n=3)$, or a combination of providing information about massage and referred to an outside entity for massage $(n=1)$.

\section{Integration Algorithm}

In order to examine the extent to which massage research is translational in cancer care, the IOM criteria were overlaid on the data and used to create an integration algorithm. A set of five variables was identified to establish an objective measurement of a level of integration. Each of the five variables was designed to reveal an optimal component of integration, and also to attempt to recognize an evolutionary process for integrating massage into cancer care (see Table 2). These variables were then used to create an integration algorithm with a 6-point range. The points were added for each of the centers to create a scale ranging from 0 to 5 .

- None (no data reported)

- Minimal (1 data point)

- Low (2 data points)

- Moderate (3 data points)

- High (4 data points)

- Very High (5 data points)
TABLE 2. Measuring Integration of Massage

Measurement Relevant to
Oncology Massage

V1. Acceptance of treatment as therapeutic

V2. Institution offers treatment to patients

V3. Clinical practice guidelines in place

V4. Evidence-based decision used to inform treatment

V5. Shared knowledge about treatment among health care team
Provide information about massage for oncology patients. Refer to outside entity that provides massage.

Massage therapy offered within the institution to cancer patients receiving treatment .

Facility has formal clinical practice guidelines for massage specifying parameters for massage treatment.

Treatment protocols or clinical approaches are predicated on evidence based-resources (i.e., research literature, text books).

Providers able to access or contribute to EMR.

Post-treatment documented in patient medical record
Centers were coded with one point for each variable meeting the applicable criteria. All of the variables were weighted equally in the algorithm creating an integration score from 0 to 5 . This recognized that any single variable when viewed alone was the equivalent of a minimal integration, while combinations of variables - in any form - were indicative of a higher level of integration.

A frequency distribution was used to apply the algorithm to the levels of integration. The integration levels of oncology massage for all 62 cancer centers ranged from None $(35.5 \%)$ to Very High $(17.7 \%)$ with distribution across five levels of integration in the algorithm. Among the 18 centers that ranked High or Very High, 16 of the centers were comprehensive cancer centers which are associated with a wholeperson approach to treatment. The majority of all of the centers in the study $(44,71.0 \%)$ achieved a level of Moderate or below indicating opportunity for improvements in integration of massage (Table 3).

The algorithm revealed an evolutionary pattern to the integration of massage. The six centers that achieved a Minimal level did so simply by providing information about massage to patients. As identified by the IOM, this is the very beginning of the integration of CAM into mainstream medical care. ${ }^{(23)}$ The 11 centers achieving a Low level of integration provided information about massage and offered massage to patients, but there was no additional evidence of integration. A slight variation was noted in the five centers achieving a Moderate level of integration: all provided information about massage and offered massage to patients, but none of the centers had formal clinical practice guidelines. A single center in 
TABLE 3. Integration Level of Massage by Center Type

\begin{tabular}{lcccccc}
\hline & \multicolumn{5}{c}{ Center Type } \\
\cline { 2 - 7 } & Clinical & $(\%)$ & Comp & (\%) & Total & $(\%)$ \\
\hline $\begin{array}{l}\text { None } \\
\text { Minimal (1 data }\end{array}$ & 3 & $12.9 \%$ & 14 & $22.6 \%$ & 22 & $35.5 \%$ \\
point) & $4.8 \%$ & 3 & $4.8 \%$ & 6 & $9.7 \%$ \\
Low (2 data points) & 2 & $3.2 \%$ & 9 & $14.5 \%$ & 11 & $17.7 \%$ \\
$\begin{array}{l}\text { Moderate (3 data } \\
\text { points) }\end{array}$ & 2 & $3.2 \%$ & 3 & $4.8 \%$ & 5 & $8.1 \%$ \\
High (4 data points) & 0 & $0.0 \%$ & 7 & $11.3 \%$ & 7 & $11.3 \%$ \\
$\begin{array}{l}\text { Very High (5 data } \\
\text { points) }\end{array}$ & 2 & $3.2 \%$ & 9 & $14.5 \%$ & 11 & $17.7 \%$ \\
TOTAL & 17 & $27.4 \%$ & 45 & $72.6 \%$ & 62 & $100.0 \%$ \\
\hline
\end{tabular}

the moderate level group drew upon evidence-based resources to guide massage treatment decisions, but did not document information about massage in the EMR. The remaining four centers documented information about massage in the EMR, but did not utilize evidence-based resources.

Variability in the integration patterns was more apparent among the seven centers that achieved a High level of integration. All of these centers provided information about massage and offered massage to patients. Even though two centers drew upon evidence-based resources to guide treatment decisions and documented massage treatments in the EMR, they lacked formal clinical practice guidelines. Formal clinical practice guidelines were in place at three centers, but did not indicate whether or not they were developed from evidence-based resources. At these three centers, treatments were documented in the EMR. The remaining two centers in this group met all of the criteria except shared knowledge: they did not document massage treatment in the EMR.

Among the 11 centers that achieved a Very High level of integration, all five levels of integration were evident: information provided about massage indicated acceptance of the therapeutic value of massage, massage was made available to patients at the cancer center, formal clinical practice guidelines were in place that were developed using evidence-based resources, and information about massage treatment was documented in the EMR creating the ability to share knowledge about massage among the health care team. Thus, at only $17.7 \%$ of all NCI-designated Cancer Centers can massage be considered to be integrated into cancer care based upon the five criteria in the algorithm.

\section{DISCUSSION}

Despite evidence that massage is a viable nonpharmacological treatment for CRS/CTRS, massage was not highly integrated at NCI-designated Cancer
Centers. This analysis revealed massage integration at a lower level than anticipated, given the number of studies on massage and CRS/CTRS. A benefit of this analysis was the creation of an integration algorithm. This serves as an important measurement to provide insight into recognition of massage for therapeutic benefits and translation of massage research into practice.

The lack of a predetermined definition of massage in this research presented the opportunity to explore what an NCI-designated Cancer Center considered as massage. The finding that two of the most frequently noted modalities (Reiki, therapeutic touch) were biofield therapies was surprising. These modalities do not involve manipulation of soft tissues and do not require a massage license to practice - but they were identified as massage by multiple centers. Identifying credentials of massage providers was beyond the scope of this study. While this presents a limitation, inclusion of modalities that are performed by non-massage therapists, may indicate that treatments are performed by other health care providers (e.g., nurses, nursing assistants). This implies recognition of the benefits of massage, but not recognition of the important role licensed massage therapists can play in cancer care.

A limitation of this research was the omission of 12 NCI-designated Cancer Centers from the survey portion of the data collection. Despite multiple attempts, it was difficult to locate individuals at these institutions to participate in the survey. Because the survey was voluntary, no specific reasons were sought for non-participation. It is worth noting, that some massage programs/providers could not be located, some individuals who were reached by phone were not willing to share information, while others were not willing to participate without financial compensation. For 9 of the 12 NCI-designated Cancer Centers that did not participate in the telephone survey, information was available on the institution's website. The broader data gathering was necessary to capture information to include a higher number of NCI-designated Cancer Centers in this study. It is worth noting that, if the research team encountered difficulty locating massage programs, patients may encounter similar problems and so may health care providers wishing to refer patients for massage.

While the algorithm presents a classification system for levels of integration that can be applied to any CAM modality, it also identifies a relatively consistent pattern to the evolution of integrative practices as a center moves from a point range of Low to Very High. To fully realize integration of massage, future research should examine characteristics associated with even higher levels of integration. A higher level of specificity could be applied by expansion of the algorithm to examine assessment and documentation of the effects of massage in general and in relation to other treatments aimed at similar CRS/CTRS (see Table 4). This includes the use of evidence-based 
TABLE 4. Possible Expansion to an 8-point Algorithm

\begin{tabular}{ll}
\hline \multicolumn{1}{c}{ Integration Variable } & \multicolumn{1}{c}{$\begin{array}{c}\text { Measurement Relevant to } \\
\text { Oncology Massage }\end{array}$} \\
\hline $\begin{array}{l}\text { V1. Acceptance of treatment } \\
\text { as therapeutic }\end{array}$ & $\begin{array}{l}\text { Provide information about } \\
\text { massage for oncology patients. } \\
\text { Refer to outside entity that } \\
\text { provides massage. }\end{array}$ \\
V2. Institution offers treatment & $\begin{array}{l}\text { Massage therapy offered within } \\
\text { the institution to cancer patients } \\
\text { to patients }\end{array}$ \\
receiving treatment
\end{tabular}

aProposed additional integration variables.

practice to inform the design of massage treatment, and implementation of informatics to track treatments and outcomes. Leveraging research to develop clinical practice guidelines specifically related to CRS/CTRS, coupled with the documentation of treatment in the EMR, would validate the importance of massage in cancer care. Expansion of the integration algorithm to include three more levels of variables would permit the opportunity to examine integration of massage at an "Excellent" or even "Fully-Integrated" level. The integration algorithm created in this analysis can also be applied to other CAM therapies and or other areas of health care, to better track inclusion and accessibility to patients.

\section{CONCLUSION}

This current study was objective and comprehensive. While it reveals a degree of integration of massage into outpatient cancer care, the results also reveals a gap in the translational area of massage research into practice. It is disappointing that $11 \mathrm{NCI}$-designated Cancer Centers provide information about massage but do not offer massage to patients. This indicates an opportunity for further dissemination of information about massage research to health care providers and administrators working in cancer care. Application of the integration algorithm to other areas of health care can reveal opportunities for outreach by the massage profession to advocate for coordinated, integrated care.

\section{ACKNOWLEDGMENTS}

This project was funded in part by a Rutgers University School of Health Professions Summer Research Grant.

\section{CONFLICT OF INTEREST NOTIFICATION}

The authors declare there are no conflicts of interest.

\section{COPYRIGHT}

Published under the CreativeCommons AttributionNonCommercial-NoDerivs 3.0 License.

\section{REFERENCES}

1. Hollen PJ, Msaouel P, Gralla RJ. Determining issues of importance for the evaluation of quality of life and patient-reported outcomes in breast cancer: results of a survey of 1072 patients. Breast Cancer Res Treat. 2015;151(3):679-686.

2. Huang IC, Hudson MM, Robison LL, Krull KR. Differential impact of symptom prevalence and chronic conditions on quality of life in cancer survivors and non-cancer individuals: a population study. Cancer Epidemiol Biomarkers Prev. 2017;26(7):1124-1132.

3. Kiely F, Cran A, Finnerty D, O'Brien T. Self-reported quality of life and symptom burden in ambulatory patients with multiple myeloma on disease-modifying treatment. Am J Hosp Palliat Care. 2017;34(7):671-676.

4. Kim Y, Yen IH, Rabow MW. Comparing symptom burden in patients with metastatic and nonmetastatic cancer. $J$ Palliat Med. 2016;19(1):64-68.

5. Rabow MW, Knish SJ. Spiritual well-being among outpatients with cancer receiving concurrent oncologic and palliative care. Support Care Cancer. 2015;23(4):919-923.

6. So WK, Marsh G, Ling WM, Leung FY, Lo JC, Yeung M, et al. The symptom cluster of fatigue, pain, anxiety, and depression and the effect on the quality of life of women receiving treatment for breast cancer: a multicenter study. Oncol Nurs Forum. 2009;36(4):E205-E214.

7. Armstrong T, Cohen MZ, Hess KR, Manning R, Lee EL, Tamavo G, et al. Complementary and alternative medicine use and quality of life in patients with primary brain tumors. J Pain Symptom Manage. 2006;32(2):148-154. 
8. Bernstein BJ, Grasso T. Prevalence of complementary and alternative medicine use in cancer patients. Oncology (Williston Park). 2001;15(10):1267-1272; discussion 1272-1268, 1283.

9. Loquai C, Dechent D, Garzarolli M, Kaatz M, Kaehler KC, Kurschat $\mathrm{P}$, et al. Use of complementary and alternative medicine: a multicenter cross-sectional study in 1089 melanoma patients. Eur J Cancer. 2017;71:70-79.

10. Sparber A, Wootton JC. Surveys of complementary and alternative medicine: Part II. Use of alternative and complementary cancer therapies. J Altern Complement Med. 2001;7(3): 281-287.

11. Yates JS, Mustian KM, Morrow GR, Gilles LJ, Padmanaban D, Atkins JN, et al. Prevalence of complementary and alternative medicine use in cancer patients during treatment. Support Care Cancer. 2005;13(10):806-811.

12. Boyd C, Crawford C, Paat CF, Price A, Xenakis L, Zhang W, the Evidence for Massage Therapy (EMT) Working Group. The impact of massage therapy on function in pain populations - a systematic review and meta-analysis of randomized controlled trials: Part II cancer pain populations. Pain Med. 2016;17(8):1553-1568.

13. Newby TA, Graff JN, Ganzini LK, McDonagh MS. Interventions that may reduce depressive symptoms among prostate cancer patients: a systematic review and meta-analysis. Psychooncology. 2015;24(12):1686-1693.

14. Somani S, Merchant S, Lalani S. A literature review about effectiveness of massage therapy for cancer pain. J Pak Med Assoc. 2013;63(11):1418-1421.

15. Lee MS, Lee EN, Ernst E. Massage therapy for breast cancer patients: a systematic review. Ann Oncol. 2011;22(6): 1459-1461.

16. Wilkinson S, Barnes K, Storey L. Massage for symptom relief in patients with cancer: systematic review. $J A d v$ Nurs. 2008;63(5):430-439.

17. Lopez G, Lee R, Sumler SS, Curtiss B, Alejandro C, Kay GM, ,et al. Impact of outpatient massage on symptom expression in cancer patients and caregivers. J Altern Complement Med. 2014;20(5):A23-A24.

18. Doherty-Gilman A. Development of a hand massage service for cancer patients undergoing chemotherapy. J Altern Complement Med. 2014;20(5):A73.

19. Running A, Seright T. Integrative oncology: managing cancer pain with complementary and alternative therapies. Curr Pain Headache Rep. 2012;16(4):325-331.

20. Harrington JE, Baker BS, Hoffman CJ. Effect of an integrated support programme on the concerns and wellbeing of women with breast cancer: a national service evaluation. Complement Ther Clin Pract. 2012;18(1):10-15.

21. Collinge W, MacDonald G, Walton T. Massage in supportive cancer care. Sem Oncol Nurs. 2012;28(1):45-54.
22. Fort DG, Herr TM, Shaw PL, Gutzman KE, Starren JB. Mapping the evolving definitions of translational research. J Clin Transl Sci. 2017;1(1):60-66.

23. Institute of Medicine. Complementary and Alternative Medicine in the United States. Washington, DC: National Academies Press; 2005.

24. Jacobs C, Graham ID, Makarski J, Chassé M, Fergusson D, Hutton B, et al. Clinical practice guidelines and consensus statements in oncology - an assessment of their methodological quality. PLoS One. 2014;9(10):e110469.

25. Smith K, Kalra D. Electronic health records in complementary and alternative medicine. Int J Med Inform. 2008;77(9): 576-588.

26. Yeo Y, Park J, Roh S, Levkoff S. Use of electronic personal health records (PHRs) for complementary and alternative medicine (CAM) disclosure: Implications for integrative health care. Complement Ther Med. 2016;26:108-116.

27. Morita T, Imura C, Fujimoto K, Shishido H, Tei Y, Inoue S. Changes in medical and nursing care in cancer patients transferred from a palliative care team to a palliative care unit. $J$ Pain Symptom Manage. 2005;29(6):595-602.

28. Carpenter WR, Meyer AM, Abernethy AP, Stürmer T, Kosorok MR. A framework for understanding cancer comparative effectiveness research data needs. J Clin Epidemiol. 2012;65(11):1150-1158.

29. Manchikanti L, Falco FJ, Benyamin RM, Helm S, 2nd, Parr AT, Hirsch JA. The impact of comparative effectiveness research on interventional pain management: Evolution from Medicare Modernization Act to Patient Protection and Affordable Care Act and the Patient-Centered Outcomes Research Institute. Pain Physician. 2011;14(3):E249-E282.

30. In H, Neville BA, Lipsitz SR, Corso KA, Weeks JC, Greenberg CC. The role of National Cancer Institute-designated cancer center status: Observed variation in surgical care depends on the level of evidence. Ann Surg. 2012;255(5):890-895.

31. Brauer JA, El Sehamy A, Metz JM, Mao JJ. Complementary and alternative medicine and supportive care at leading cancer centers: a systematic analysis of websites. J Altern Complement Med. 2010;16(2):183-186.

32. Hamad EO, Savundranayagam MY, Holmes JD, Kinsella EA, Johnson AM. Toward a mixed-methods research approach to content analysis in the digital age: the combined contentanalysis model and its applications to health care Twitter feeds. J Med Internet Res. 2016;18(3):e60.

Corresponding author: Virginia S. Cowen, PhD, LMT, Department of Primary Care, Rutgers School of Health Professionals, 65 Bergen St., Newark, NJ 07107-1709, USA

E-mail: gscowen@msn.com 